\title{
Embracing instability
}

MYC and NMYC are notorious oncoproteins with surprisingly short half-lives. Could this be the key to their downfall in tumour cells and to that of the tumour?

One of the ubiquitin ligases involved in the degradation of MYC proteins is the FBXW7-directed SKP1-CUL1-F-box (SCF) complex. One allele of $F B X W 7$ is often mutated in T cell acute lymphoblastic leukaemia (T-ALL), and Iannis Aifantis and colleagues investigated the function of a common mutant, FBXW7 $7^{\mathrm{R} 465 \mathrm{C}}$ (FBXW7 $7^{\mathrm{R} 468 \mathrm{C}}$ in mice). To test the effect of mutant FBXW7 in leukaemic cells, bone marrow cells with mutant Fbxw $7, F b x w 7^{+/-}$or wild-type Fbxw 7 were infected with a retrovirus expressing NOTCH1 $\triangle \mathrm{E}$, a NOTCH1 mutant that functions similarly to a frequently found NOTCH1 mutation in human T-ALL samples with FBXW7 ${ }^{\mathrm{R} 465 \mathrm{C}}$ mutations. Mice transplanted with bone marrow cells that expressed both mutant FBXW7 and NOTCH1 $\triangle \mathrm{E}$ developed leukaemia more rapidly than mice transplanted with cells that expressed NOTCH1 $\triangle \mathrm{E}$ and that were wild type or heterozygous for Fbxw7. The leukaemic blasts expressing mutant FBXW7 had an immature $\mathrm{T}$ cell phenotype and efficiently established disease on transplantation, prompting the authors to examine the leukaemiainitiating cell (LIC) population. This cell population was tenfold higher in FBXW7-mutant T-ALL than in FBXW7 wild-type T-ALL. Moreover, LICs also expressed increased levels of MYC, and the authors confirmed in vitro that mutant FBXW7 was unable to bind and ubiquitylate MYC. The treatment of mouse T-ALL with CPI203, a derivative of the bromo and extra terminal (BET) protein inhibitor JQ1 that is known to reduce
MYC levels, reduced the leukaemic burden. High-throughput RNA sequencing indicated that BET protein inhibition reduced the expression of genes that were upregulated as a result of increased MYC levels in the context of mutant FBXW7 and NOTCH activity. These findings indicate that targeting MYC in T-ALL might lead to the loss of the LIC population.

However, the MYC family of proteins is not easy to target with small molecules. Louis Chesler, Martin Eilers and colleagues asked whether disrupting the association between NMYC and Aurora kinase A (AURKA) - NMYC binding to AURKA prevents NMYC ubiquitylation by FBXW7 during mitosis - would have a therapeutic effect in neuroblastoma. The authors investigated an AURKA inhibitor, MLN8054, which causes a substantial alteration in the conformation of the kinase domain that binds NMYC. Using MLN8054 and a related compound, MLN8237, to treat neuroblastoma cell lines with amplified $M Y C N$ resulted in reduced levels of NMYC, but not in reduced $M Y C N$ mRNA levels. Moreover, treatment for 24 hours resulted in $70 \%$ of the cells arresting in the G2/M phase of the cell cycle and accelerated turnover of $\sim 70 \%$ of the NMYC protein. Importantly, proximity ligation assays showed that both drugs disrupted the complex between NMYC and AURKA.
So, are MLN8054 and MLN8237 potential therapies for NMYCdriven neuroblastoma? The treatment of mice with an aggressive $M Y C N$ driven neuroblastoma with the MLN compounds resulted in tumour shrinkage, with $~ 50 \%$ complete response rates (in terms of tumour volume) for both compounds and increased survival. In addition, gene set enrichment analyses showed that the genes that were altered as a result of MLN8054 treatment and NMYC degradation correlated with overall and event-free survival in 476 primary patient samples. Moreover, these data also indicate that MLN8054 downregulates genes that are associated with pluripotent stem cells, and further analyses suggest that MLN8054 limits the self-renewal of neuroblastoma cells and induces differentiation.

Thus, both of these papers indicate that increasing the turnover of MYC proteins might promote the loss of an immature, self-renewing cell population and might, therefore, have a therapeutic impact.

Nicola McCarthy

ORIGINAL RESEARCH PAPERS King, B. et al.

The ubiquitin ligase FBXW7 modulates

leukemia-initiating cell activity by regulating MYC stability. Cell 153, 1552-1566 (2013)| Brockmann, M. et al. Small molecule inhibitors of Aurora-A induce proteasomal degradation of

$\mathrm{N}-\mathrm{Myc}$ in childhood neuroblastoma. Cancer Cell

http://dx.doi.org/10.1016/j.ccr.2013.05.005 (2013) 